\title{
A Comparison of Proximal and Distal High-Frequency Jet Ventilation in an Experimental Animal Model
}

\author{
Kenneth P. Bandy, RRT, Steven M. Donn, MD, ${ }^{\dagger}$ Joanne J. Nicks, RRT,* and Ronald A. Naglie, MD ${ }^{\dagger}$
}

\begin{abstract}
Summary. High-frequency jet ventilation using either a proximal or a distal endotracheal injection site through a triple-lumen endotracheal tube was studied in 10 adult cats. The comparative effects on pulmonary gas exchange, tracheal pressure, heart rate, and blood pressure were examined for each injection site at both high (8-12 pounds per square inch [PSI] and low (5-8 PSI) jet-driving pressures in normal and lung-injured cats. Lung injury was created by modification of a surfactant washout technique previously demonstrated in rabbits. Alveolar ventilation $\left(\mathrm{PaCO}_{2}\right)$ was found to be significantly better with distal than with proximal jet injection under all experimental conditions. At high jet-driving pressures, peak inspiratory pressure was higher in both normal $(p=0.03)$ and lung-injured cats ( $p$ $=0.002$ ) with distal high-frequency jet ventilation. In addition, lung-injured animals were observed to have higher distal mean airway pressures at high jet-driving pressures $(p<$ $0.01)$. No differences in oxygenation were found in any circumstances. The results of this animal study suggest that distal high-frequency jet ventilation may be more effective in those situations in which improvement in alveolar ventilation is the major goal and that during proximal high-frequency jet ventilation airway pressures should be monitored as far distally as possible. (Key words: high-frequency jet ventilation; distal versus proximal jet injection; artificial lung injury; airway pressure; monitoring sites; cats.) Pediatr Pulmonol 1986; 2:225-229.
\end{abstract}

Over the past several years the study of highfrequency jet ventilation (HFJV) and its application to neonatal respiratory disease ${ }^{1-3}$ has received considerable attention. Investigations exploring the additional applications of HFJV describe the placement of the jet injection site at the distal end of either a commercially available triple-lumen endotracheal tube ${ }^{4-7}$ or a modified standard single-lumen endotracheal tube. ${ }^{8}$ Other studies have examined the effects of placing the jet injection site at the proximal end of the endotracheal tube..$^{1-3}$ Several investigations have also described the effects of the endotracheal tube and jet placement on air entrainment and jet flow during HFJV. ${ }^{7,9}$ These investigations on the interaction of jet placement. air entrainment, and the endotracheal tube itself have been only fragmentarily reported, and there is virtually no documentation of the physiologic re-

From the Department of Pediatric Respiratory Therapy* and the Department of Pediatrics, Section of Newborn Services, ${ }^{\dagger}$ University of Michigan Medical Center, Ann Arbor, Michigan.

Received November 22, 1985; revision accepted for publication March 7, 1986

Presented in part at the Second Conference on High Frequency Ventilation of the Newborn. Park City, Utah, April 2-4, 1985.

Address correspondence and reprint requests to Dr. Donn: University of Michigan Medical Center, Department of Pediatrics-Newborn Services, L3023 Women's Hospital, Box 0254, 1500 East Medical Center Drive, Ann Arbor, Michigan 48109-0254. sponse to the variation of the jet injection site during HFJV.

The purpose of this study was to document differences in proximal and distal tracheal pressures during proximal injection HFJV and to determine differences in pulmonary gas exchange, tracheal pressure, heart rate, and blood pressure when using proximal or distal jet injection sites. An additional objective of this study was to create an animal model of surfactant deficiency in the adult cat by adapting a saline lavage technique previously demonstrated in rabbits. ${ }^{10}$

\section{Methods}

Ten adult cats weighing from 2 to $4 \mathrm{~kg}$ were anesthesized with pentobarbital $(25-30 \mathrm{mg} / \mathrm{kg})$. The animals were intubated with a "Hi-Lo" Jet Tracheal Tube (National Catheter Company) of 3.0 or $4.0 \mathrm{~mm}$ I.D. Arterial access was established by cannulation of the right internal carotid artery with a 3.5-Fr arterial catheter. Heart rate and blood pressure were measured using a Tektronix 413A Neonatal Monitor with a Gould P23ID Pressure Transducer. Sequential blood gas analyses were performed using a Radiometer ABL 30 Blood Gas Analyzer. Ventilation was provided by using the Sechrist 990 High Frequency Jet Ventilator. This high-frequency 


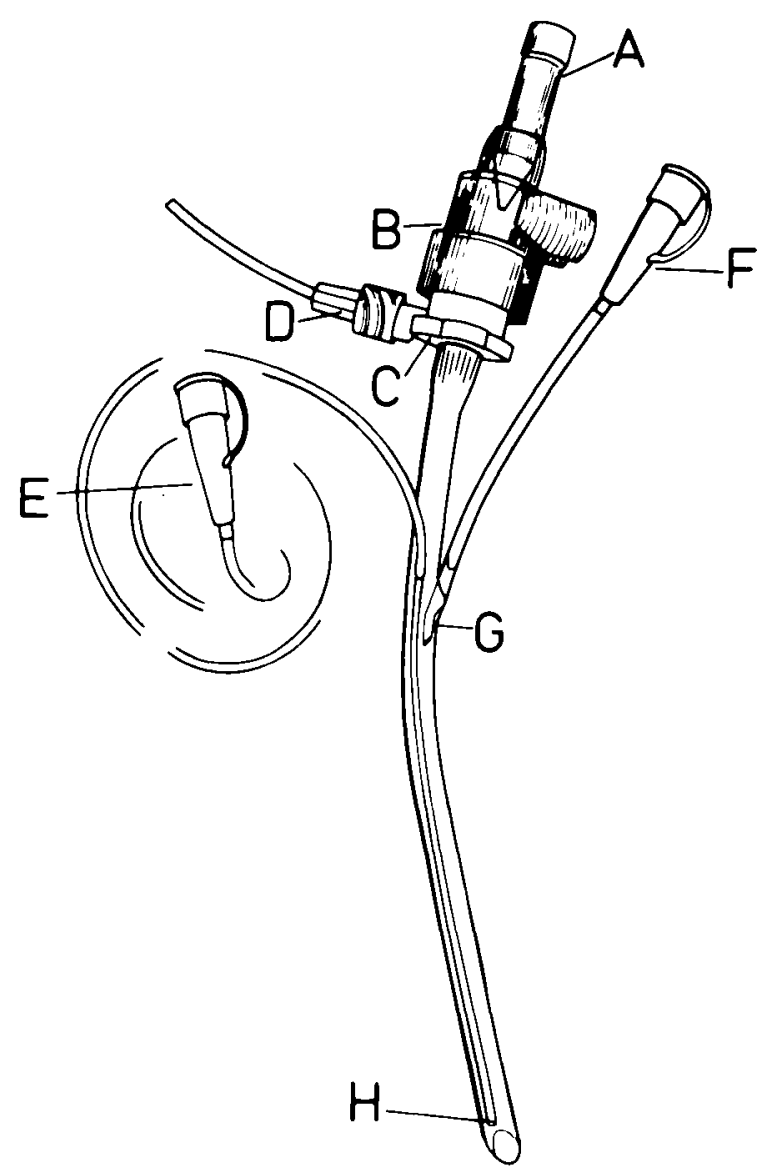

figure 1-Schematic diagram of triple-lumen endotracheal tube and adapter. A. Proximal jet injector. B. Patient connector. C. Adapter. D. Proximal pressure monitoring port. E. Distal pressure monitoring port. F. Distal jet injection connector. $G$. Distal jet injection site. $H$. Distal pressure monitoring site.

jet ventilator is a solenoid-driven, pulse-flow, time-cycled positive pressure ventilator that delivers a volume of gas at a controlled pressure and a fractional concentration of oxygen either to a jet adapter placed at the proximal end of the endotracheal tube or to the appropriate jet injection lumen of the "Hi-Lo" Jet Tracheal Tube (figure 1). The proximal injection cannula is a tapered device with a distal diameter of $0.7 \mathrm{~mm}$. The distal injection cannula has a diameter of $2.0 \mathrm{~mm}$.

The initial ventilator settings were arbitrarily fixed at an $\mathrm{FlO}_{2}$ of 1.0 , a frequency of 300 breaths per minute, an inspiratory fraction of $33 \%$, and a positive end expiratory pressure of 0 . For these initial settings, the jet-driving pressure was adjusted in order to reach a eucapnic state. Tracheal pressures were measured using the high-frequency response pressure transducers in the Sechrist 990 High Frequency Jet Ventilator. These have been described previ- ously. ${ }^{3}$ Proximal pressure measurements were obtained $2 \mathrm{~cm}$ beyond the jet injection site. Distal pressure measurements were obtained from within the appropriate endotracheal tube, 1.0 $\mathrm{cm}$ above its distal tip.

The study design consisted of two phases for both normal and lung-injured animals (figure 2). During phase I the animals were ventilated to a eucapnic state $\left(\mathrm{PCO}_{2}=40 \pm 5\right.$ torr $)$. Each animal was then randomly selected to receive either proximal or distal jet injection for the initial mode. Blood gases, heart rate, blood pressure, and tracheal pressures were recorded 30 minutes later. Each animal was then venti. lated with the alternative jet injection site, and measurements were repeated in 30 minutes. Phase II included measurements obtained following an increase in the jet-driving pressure to a level that was $50 \%$ greater than that in phase I. The protocol was then repeated with measurements obtained every 30 minutes for 2 hours. During use of the proximal jet injection site dual pressure recordings were obtained from both the proximal and the distal endotracheal monitor. ing sites.

Following completion of these studies in the normal animals, the lung injury was induced by a surfactant washout technique adapted from

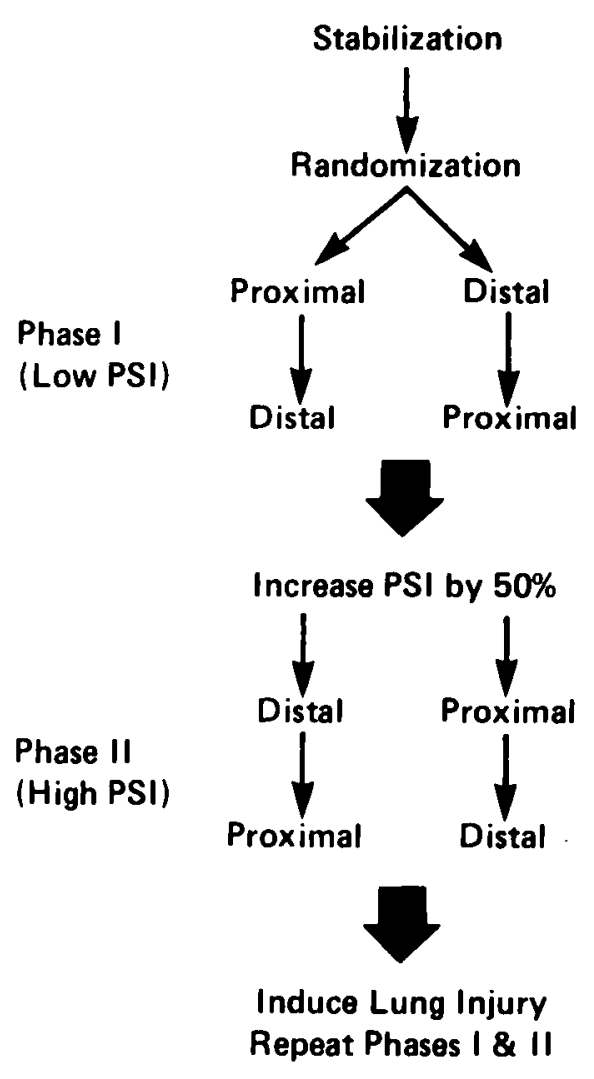

figure 2-Study protocol. 
table 1-Comparison of Proximal and Distal Tracheal Pressures During Proximal HFJ in Normal Cats $(N=10)$

\begin{tabular}{|c|c|c|c|}
\hline & $\begin{array}{c}\text { Proximal } \\
\text { Tracheal Pressure } \\
\left(\mathrm{Cm} \mathrm{H}_{2} \mathrm{O}\right)\end{array}$ & 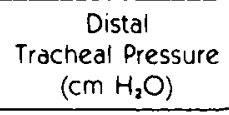 & $P$ \\
\hline \multicolumn{4}{|c|}{ Low (5-8 PSI) driving pressure } \\
\hline PIP & $3.6 \pm 1.5^{*}$ & $2.2 \pm 1.0$ & 0.02 \\
\hline Pay & $1.3 \pm 0.5$ & $1.0 \pm 0.5$ & NS \\
\hline \multicolumn{4}{|c|}{ High (8-12 PSI) driving pressure } \\
\hline$P \mid P$ & $6.4 \pm 2.1$ & $2.9 \pm 0.7$ & 0.001 \\
\hline Pâw & $2.0 \pm 0.4$ & $1.3 \pm 0.4$ & 0.0012 \\
\hline
\end{tabular}

previous work in rabbits. ${ }^{10}$ The cats were subjected to bilateral pulmonary lavage utilizing $0.9 \%$ sodium chloride prewarmed to $37^{\circ} \mathrm{C}$. A volume of $25 \mathrm{ml} / \mathrm{kg}$ of body weight was instilled into the trachea at pressures no greater than $40 \mathrm{~cm}$ of water over a 20 -second period and repeated at 5 minute intervals. Following instillation, the animals were suctioned and received bagged ventilation with $100 \%$ oxygen. Pulmonary lavage was continued until either the $\mathrm{PaO}_{2}$ dropped to less than 50 torr or a maximum of 10 lavages had been performed. In order to validate surfactant removal, tracheal aspirates were randomly chosen from four cats and were subjected to determination of lecithin-sphingomyelin ratio. After establishment of lung injury the phase I and phase II protocols were repeated.

Data were analyzed by paired, two-sided Student's t tests, and significance was achieved if $p<0.05$. The tabulated values represent the means and SDs of every measurement obtained during each phase.

\section{Results}

\section{Creation of Lung Injury}

Tracheal aspirates demonstrated lecithinsphingomyelin rations of $5.02: 1$ to $7.94: 1$, indicating washout of pulmonary surfactant. Alveolar instability was further demonstrated by marked increases in the $\mathrm{P}(\mathrm{A}-\mathrm{a})_{\mathrm{O}_{2}}$ from mean 148 torr before to mean 628 torr after lung lavage at both low and high jet-driving pressures. All 10 animals survived the duration of the experimental procedure in stable physiologic states.

\section{Proximal and Tracheal Pressures During Proximal HFJV}

Table 1 demonstrates the comparison of the proximal and distal tracheal pressures during use of the proximal jet injection site. In normal animals, receiving low jet-driving pressures, a statistically significant difference between peak pressures was recorded at the proximal and distal endotracheal tube sites, with higher peak inspiratory pressure at the proximal site. However. there were no statistically significant differences in mean airway pressure between the sites. At an increased jet-driving pressure, statistically significant differences were found between the proximal and distal sites for both inspiratory pressure and mean airway pressure.

Table 2 demonstrates the comparison of proximal and distal tracheal pressures during proximal-injection high-frequency jet ventilation in the lung-injured cats. When the low jetdriving pressures were used, there were no significant differences between pressures measured proximally and distally for either the inspiratory pressure or the mean airway pressure. However, when the driving pressure was increased, statistically significant differences were found. Both the peak inspiratory pressure and the mean airway pressure were found to be sig. nificantly greater at the proximal end of the endotracheal tube compared with the distal end.

\section{Comparison of Proximal and Distal HFJV}

In animals with normal lungs, low jet-driving pressure was associated only with an improvement in ventilation, when the distal jet injection site was used (table 3 ). When jet-driving pressure was increased, these cats also showed a significantly higher peak inspiratory pressure and heart rate with use of the distal jet injection site (table 4).

Creation of lung injury by surfactant washout resulted in nearly identical changes. At low jetdriving pressures (table 5 ), the only significant change between the two injection sites was a marked reduction in $\mathrm{P}_{\mathrm{CO}_{2}}$ with use of distal injection. When jet-driving pressures were in- 
table 2-Comparison of Proximal and Distal Tracheal Pressures During Proximal HFJV in Lung-Injured Cats $(N=10)$

\begin{tabular}{|c|c|c|c|}
\hline & $\begin{array}{c}\text { Proximal } \\
\text { Tracheal Pressure } \\
\left(\mathrm{cm} \mathrm{H}_{2} \mathrm{O}\right) \\
\end{array}$ & $\begin{array}{c}\text { Distal } \\
\text { Tracheal Pressure } \\
\left(\mathrm{Cm} \mathrm{H} \mathrm{H}_{2} \mathrm{O}\right) \\
\end{array}$ & $p$ \\
\hline \multicolumn{4}{|c|}{ Low (5-8 PSI) driving pressure } \\
\hline PIP & $11.0 \pm 7.3^{*}$ & $7.5 \pm 3.5$ & NS \\
\hline Pâw & $3.2 \pm 1.8$ & $2.4 \pm 0.8$ & NS \\
\hline \multicolumn{4}{|c|}{ High (8-12 PSI) driving pressure } \\
\hline PIP & $19.4 \pm 7.2$ & $8.1 \pm 2.6$ & 0.0002 \\
\hline Paw & $5.6 \pm 2.1$ & $2.9 \pm 0.8$ & 0.001 \\
\hline
\end{tabular}

$P \mid P=$ peak inspiratory pressure

Pāw = mean airway pressure.

PSI = pounds per square inch

NS $=$ not significant.

- Values are mean \pm SD (for explanation see under Methods)

creased, use of the distal injection site resulted in significant increases in peak inspiratory pressure, mean airway pressure, and ventilation (table 6).

\section{Discussion}

The results of this study suggest that significantly higher peak pressures exist at the proximal monitoring site than at the distal monitoring site during proximal-injection HFJV. This difference is accentuated at higher jet-driving pressures. In animals with normal lungs, these differences are greater for peak inspiratory pressure than for mean airway pressure. However, in animals with lung injury, differences are significant only when higher jet-driving pressures are used. It appears that endotracheal tube resistance may play a major factor in dissipating intratracheal pressure during proximal-injection HFJV, under conditions of normal lung compliance. However, when lung compliance is reduced following removal of surfactant, this effect

table 3-Comparison of Proximal and Distal Injection HFJV in Normal Cats at Low Jet Driving Pressure (5-8 PSI), $N=10$

\begin{tabular}{lccc}
\hline & $\begin{array}{c}\text { Proximal } \\
\text { Tracheal Pressure }\end{array}$ & $\begin{array}{c}\text { Distal } \\
\text { Tracheal Pressure }\end{array}$ & p \\
\hline $\mathrm{PIP}\left(\mathrm{cm} \mathrm{H} \mathrm{H}_{2} \mathrm{O}\right)$ & $2.2 \pm 1.0^{*}$ & $3.1 \pm 1.1$ & NS \\
Pä $\left(\mathrm{cm} \mathrm{H}_{2} \mathrm{O}\right)$ & $1.0 \pm 0.5$ & $1.3 \pm 0.6$ & NS \\
$\mathrm{P}_{\mathrm{CO}_{2} \text { (torr }}$ & $46.4 \pm 10.2$ & $34.2 \pm 7.7$ & 0.007 \\
$\mathrm{PO}_{2}(\mathrm{torr})$ & $510.3 \pm 65.3$ & $530.7 \pm 41.3$ & $\mathrm{NS}$ \\
$\mathrm{HR}(\mathrm{bpm})$ & $109.2 \pm 10.5$ & $105.4 \pm 6.7$ & NS \\
MAP $(\mathrm{mm} \mathrm{Hg})$ & $70.1 \pm 11.7$ & $75.2 \pm 13.4$ & NS \\
\hline
\end{tabular}

PSI = pounds per square inch.

$P I P=$ peak inspiratory pressure

Paw = mean airway pressure .

$\mathrm{PCO}_{2}=$ arterial carbon dioxide tension.

$\mathrm{PO}_{2}=$ arterial oxygen tension.

$H R=$ heart rate.

bpm $=$ beats per minute

$M A P=$ mean arterial pressure

NS = not significant.

- Values are mean \pm SD (for explanation see under Methods). is lessened. Some artifact in the measurement of pressures might be expected because of differing gas flow rates at the proximal and distal sites. We attempted to minimize this effect by using an endotracheal tube with the smallest possible diameter. The variability in pressure recordings obtained during proximal-injection HFJV indicates the need for uniformity of pressure transducer placement among various investigations of HFJV.

The comparisons of proximal and distal HFJV injection sites may also be of clinical significance. Under all experimental conditions more effective ventilation was achieved when the distal injection site was utilized. This was especially true at higher jet driving pressures, resulting in greater peak inspiratory pressure in both normal and lung-injured cats. No apparent differences in oxygenation were observed under any experimental circumstances. The more effective ventilation with distal HFJV may be related to a higher peak inspiratory pressure (and pressure amplitude change) compared with

table 4-Comparison of Proximal and Distal Injection HFJV in Normal Cats at High Jet-Driving Pressure (8-12 PSI), $N=10$

\begin{tabular}{lccc}
\hline & $\begin{array}{c}\text { Proximal } \\
\text { Injection }\end{array}$ & $\begin{array}{c}\text { Distal } \\
\text { Injection }\end{array}$ & $\mathrm{P}$ \\
\hline $\mathrm{PIP}\left(\mathrm{cm} \mathrm{H} \mathrm{H}_{2} \mathrm{O}\right)$ & $2.9 \pm 0.7^{*}$ & $3.7 \pm 0.8$ & 0.03 \\
$\mathrm{PaW}\left(\mathrm{cm} \mathrm{H}_{2} \mathrm{O}\right)$ & $1.3 \pm 0.4$ & $1.7 \pm 0.4$ & $\mathrm{NS}$ \\
$\mathrm{PCO}_{2}$ (torr) & $36.0 \pm 7.9$ & $16.0 \pm 3.7$ & $<0.00001$ \\
$\mathrm{PO}_{2}$ (torr) & $548.6 \pm 64.3$ & $536.1 \pm 69.7$ & $\mathrm{NS}$ \\
$\mathrm{HR}(\mathrm{bpm})$ & $96.8 \pm 7.1$ & $104.1 \pm 7.5$ & 0.03 \\
$\mathrm{MAP}(\mathrm{mm} \mathrm{Hg})$ & $73.4 \pm 13.0$ & $62.1 \pm 22.3$ & $\mathrm{NS}$ \\
\hline
\end{tabular}

PSI = pounds per square inch .

PIP = peak inspiratory pressure.

Paw $=$ mean airway pressure.

$\mathrm{PCO}_{2}=$ arterial carbon dioxide tension.

$\mathrm{PO}_{2}=$ arterial oxygen tension.

$\mathrm{HR}=$ heart rate

bom $=$ beats per minute

MAP = mean arterial pressure

NS $=$ not significant.

- Values are mean \pm SD (for explanation see under Methods) 
table 5-Comparison of Proximal and Distal Injection HFJV in Lung-Injured Cats at Low Jet-Driving Pressure (6-14 PSI),

$$
N=10
$$

\begin{tabular}{lccc}
\hline & $\begin{array}{c}\text { Proximal } \\
\text { Injection }\end{array}$ & $\begin{array}{c}\text { Distal } \\
\text { Injection }\end{array}$ & $\mathrm{p}$ \\
\hline $\mathrm{PIP}\left(\mathrm{cm} \mathrm{H} \mathrm{H}_{2} \mathrm{O}\right)$ & $7.5 \pm 3.5^{*}$ & $9.3 \pm 4.5$ & $\mathrm{NS}$ \\
$\mathrm{PäW}\left(\mathrm{CmH}_{2} \mathrm{O}\right)$ & $2.4 \pm 0.8$ & $3.3 \pm 1.5$ & $\mathrm{NS}$ \\
$\mathrm{PCO}_{2}(\mathrm{torr})$ & $46.2 \pm 10.0$ & $28.8 \pm 9.2$ & 0.0008 \\
$\mathrm{PO}_{2}(\mathrm{torr})$ & $46.1 \pm 12.4$ & $48.0 \pm 11.6$ & $\mathrm{NS}$ \\
$\mathrm{HR}(\mathrm{bpm})$ & $108.1 \pm 18.0$ & $95.2 \pm 16.5$ & $\mathrm{NS}$ \\
$\mathrm{MAP}(\mathrm{mm} \mathrm{HS})$ & $87.3 \pm 18.2$ & $76.3 \pm 19.2$ & $\mathrm{NS}$ \\
\hline
\end{tabular}

$P S 1=$ pounds per square inch

$P I P=$ peak inspiratory pressure

Paw $=$ mean airway pressure

$\mathrm{PCO}_{2}=$ arterial carbon dioxide tension

$\mathrm{PO}_{2}=$ arterial oxygen tension.

$H R=$ heart rate.

bPm = beats per minute.

MAP = mean arterial pressure

NS = not significant

- Values are mean \pm SD (for explanation see under Methods)

proximal-injection HFJV. The results may or may not have been similar under experimental conditions in which ventilator manipulations were allowed in order to match either pressures or arterial blood gases achieved by the two modes of HFJV. However, such comparisons were not among our present study objectives.

Creation of a model of alveolar instability in the cat has been demonstrated. The original description of the surfactant washout technique referred to the rabbit. ${ }^{10}$ We have found rabbits to be extremely labile during experiments with HFJV, particularly with respect to heart rate and blood pressure. This problem was not observed in cats. Moreover, use of the cat allowed direct laryngoscopy and intubation and obviated the need for tracheostomy.

The results of this animal study suggest that distal-injection HFJV may be more effective than proximal-injection HFJV in situations in which improved ventilation is the major therapeutic goal. Our results corroborate the finding of Frantz and Close, who determined that proximal monitoring of airway pressures inadequately estimated mean airway pressure or alveolar pressure swings during HFJV in rabbits and that tracheal pressure was more indicative of alveolar pressure." Thus, during HFJV airway pressure measurements should be monitored as far distally as possible. We believe it is imperative that a uniform system of pressure monitoring and reporting be developed in order to allow meaningful comparisons between different investigations of HFJV. table 6-Comparison of Proximal and Distal Injection HFJV in Lung-Injured Cats at High Jet-Driving Pressure (9-18 PSI),

$$
N=10
$$

\begin{tabular}{lccc}
\hline & $\begin{array}{c}\text { Proximal } \\
\text { Injection }\end{array}$ & $\begin{array}{c}\text { Distal } \\
\text { Injection }\end{array}$ & p \\
\hline PIP $\left(\mathrm{Cm} \mathrm{H}_{2} \mathrm{O}\right)$ & $8.1 \pm 2.6^{*}$ & $12.6 \pm 3.1$ & 0.002 \\
$\mathrm{PaW}\left(\mathrm{Cm} \mathrm{H}_{2} \mathrm{O}\right)$ & $2.9 \pm 0.8$ & $4.6 \pm 1.7$ & 0.01 \\
$\mathrm{P}_{\left.\mathrm{CO}_{2} \text { (torr }\right)}$ & $27.4 \pm 14.2$ & $13.5 \pm 5.5$ & 0.01 \\
$\mathrm{P}_{2}$ (torr) & $52.5 \pm 12.3$ & $76.4 \pm 55.8$ & NS \\
HR (bpm) & $89.0 \pm 15.5$ & $88.3 \pm 13.2$ & NS \\
MAP (mm H9) & $72.3 \pm 16.5$ & $60.1 \pm 18.9$ & NS \\
\hline
\end{tabular}

$P S I=$ pounds per square inch

$P I P=$ peak inspiratory pressure

Paw = mean airway pressure.

$\mathrm{PCO}_{2}=$ arterial carbon dioxide tension

$\mathrm{PO}_{2}=$ arterial oxygen tension

$H R=$ heart rate

bom $=$ beats per minute

MAP = mean arterial pressure

NS = not significant.

- Values are mean \pm SD (for explanation see under Methods)

We thank the Sechrist Corporation for providing the ventilator used in this study; K. M. J. Menon, Ph.D., for performing the lecithin. sphingomyelin ratios; and the numerous pediatric respiratory therapists who participated in this project.

\section{References}

1. Pokora $T$, Bing D, Mammel $M$, Boros $S$. Neonatal high-frequency jet ventilation. Pediatrics 1983; 72:27-32.

2. Carlo WA, Chatburn RL, Martin RJ, et al. Decrease in airway pressure during high-frequency jet ventilation in infants with resplratory distress syndrome. J Pediatr 1984; 104:101-107.

3. Donn SM, Nicks لע, Bandy KP, Naglie RN. Proximal high-frequency jet ventilation of the newborn. Pediatr Pulmonol 1985; 1:267-271.

4. Paloski WH, Barie PS, Mullins R, Newell JC. Effects of changing inspiratory to expiratory time ratio on carbon dioxide elimination during high-frequency jet ventilation. Am Rev Respir Dis 1985; 131:109-114.

5. Guntupalli K, Sladen A, Klain M. High-frequency jet ventilation and tracheobronchial suctioning. Crit Care Med 1984; 12:791-792.

6. Schuster DP, Klain M, Snyder JV. Comparison of high-frequency jet ventilation to conventional ventilation during severe acute respiratory failure in humans. Crit Care Med 1982; 10:625-630.

7. Hamilton $\mathrm{LH}$, Londino $\mathrm{JM}$, Linehan $\mathrm{JH}, \mathrm{Neu} J$. Pediatric endotracheal tube designed for high-frequency ventilation. Crit Care Med 1984; 12:988-993

8. Takahashi $H$, Takezawa J, Nishijima MK, Shindoh $Y$, et al. Effects of driving pressure and respiratory rate on airway pressure and $\mathrm{PaCO}_{2}$ in rabbits during high frequency jet ventilation. Crit Care Med 1985; 13:728-732.

9. Miodownik S, Ray C, Carlon G, et al. High frequency jet ventilation: Technical implications. Crit Care Med 1984; 12:718-720.

10. Lachmann BR, Vogel IJ. In vivo lung lavage as an experimental model of the respiratory distress syndrome. Acta Anaesthesiol 1984; $24: 231-236$

11. Frantz ID, Close RH. Elevated lung volume and alveolar pressure during jet ventilation of rabbits. Am Rev Respir Dis 1985; 131:134-138. 